\title{
ARTICLE \\ Epidemiology \\ Cigarette smoking and thyroid cancer risk: a cohort study
}

Ara Cho ${ }^{1,2}$, Yoosoo Chang ${ }^{1,3,4}$, Jiin Ahn ${ }^{1}$, Hocheol Shin ${ }^{1,5}$ and Seungho Ryu ${ }^{1,3,4}$

BACKGROUND: We evaluated the association between smoking status and thyroid cancer risk and whether this association is mediated by body mass index (BMI) and thyroid-stimulating hormone (TSH).

METHODS: We performed a cohort study of 96,855 Korean adults who were followed annually or biennially for a median of 5.9 years. RESULTS: During 511,052.9 person-years of follow-up, 1,250 participants developed thyroid cancer. In men, we observed a dosedependent inverse association between current smoking, pack-years, and thyroid cancer. After adjustment for confounders, adjusted hazard ratios (95\% confidence intervals) for thyroid cancer comparing current and former smokers to never smokers were $0.58(0.45-0.75)$ and $0.93(0.73-1.18)$, respectively. After further adjustment for BMI and TSH as potential mediators, this association was slightly attenuated, but remained significant. For women, current smokers tended to have a lower risk of thyroid cancer, but this association did not reach statistical significance.

CONCLUSIONS: In this cohort study, current smoking was associated with a decreased risk of incident thyroid cancer in men but not in women and this association was observed even after adjusting for TSH and BMI levels as potential mediators. Further mechanistic studies are needed to elucidate the possible effect of smoking on the pathogenesis of thyroid cancer development.

British Journal of Cancer (2018) 119:638-645; https://doi.org/10.1038/s41416-018-0224-5

\section{INTRODUCTION}

The incidence of thyroid cancer has increased substantially over the past few decades in most areas of the world. ${ }^{1,2}$ This increase can be attributed to enhanced detection of small, clinically insignificant thyroid tumors through increasingly widespread use of sensitive screening and diagnostic procedures. ${ }^{3,4}$ Besides the contribution of over-diagnosis, evidence also indicates that a true increase is also occurring. ${ }^{4}$ Some factors such as ionizing radiation exposure, female sex, non-cancerous benign thyroid conditions, and obesity appear to increase the risk of thyroid cancer, but the causes of thyroid cancer are not fully understood. ${ }^{4}$

Smoking is the single greatest avoidable risk factor for various types of cancer, including acute myeloid leukemia, lung, esophagus, larynx, mouth, throat, kidney, bladder, stomach, cervix, and colorectal cancers. ${ }^{5}$ The association between cigarette smoking and thyroid cancer has been evaluated in observational studies, but the results remain controversial. Some case-control ${ }^{6-16}$ and cohort studies showed an inverse relationship between smoking and thyroid cancer, ${ }^{17-20}$ while other studies found no association. ${ }^{21-23}$ Recently, in a meta-analysis of 25 case-control studies and six cohort studies, smoking was associated with a lower risk of thyroid cancer in current smokers, but this association was observed only in casecontrol studies, requiring a well-planned cohort study with a larger sample size. ${ }^{24}$ Additionally, in previous studies, the mechanism underlying the inverse association between smoking and thyroid cancer has been proposed to be mediated by lower BMI and lower thyroid-stimulating hormone (TSH) in smokers. ${ }^{25}$ Indeed, smoking has been reported to be associated with reduced body weight, leading to a lower $\mathrm{BMI}^{25,26}$ Smoking appears to be associated with adverse effects on the thyroid, resulting in changes in circulating hormone concentrations, while higher TSH values are associated with a higher frequency and more advanced stages of thyroid cancer. ${ }^{27,28}$ Until now, no cohort studies have evaluated the effect of smoking on the incidence of thyroid cancer while taking into account the effects of $\mathrm{BMI}$ and $\mathrm{TSH}$.

Therefore, we performed a longitudinal cohort study to evaluate the association between smoking status and the development of thyroid cancer in young and middle aged Korean men and women and examined whether this association was mediated by TSH levels and BMI.

\section{METHODS}

Study population

The Kangbuk Samsung Health Study is a retrospective cohort study of men and women 18 years of age or older who underwent

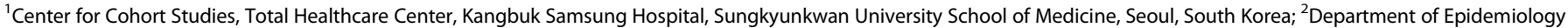

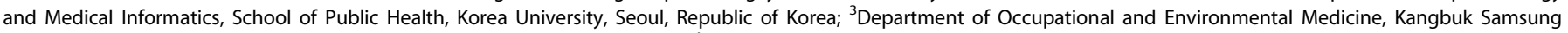

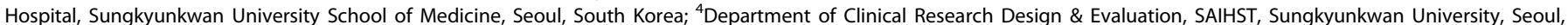
South Korea and ${ }^{5}$ Department of Family Medicine, Kangbuk Samsung Hospital, Sungkyunkwan University School of Medicine, Seoul, South Korea Correspondence: Yoosoo Chang (yoosoo.chang@gmail.com) or Seungho Ryu (sh703.yoo@gmail.com)
} 
a comprehensive annual or biennial health examination at the clinics of the Kangbuk Samsung Hospital Total Healthcare Screening Center in Seoul and Suwon, South Korea, from 2002 to present. ${ }^{29,30}$ Most examinees (over $80 \%$ ) are employees of various companies or local governmental organizations and their spouses. In South Korea, the Industrial Safety and Health Law requires annual or biennial health screening examinations of all employees, free of charge. The rest of the examinees voluntarily purchased health screening exams at the healthcare center.

The present study used de-identified retrospective data routinely collected during the health screening process of all study participants with at least one follow-up visit who underwent a comprehensive health examination at clinics between 2006 and 2008 and were followed annually or biennially until December $2014(N=98,553)$. A total of 1,698 participants were excluded due to missing data on smoking status, TSH levels, or BMI $(n=264)$, or a history of cancer $(n=1,437)$. Because some individuals met more than one exclusion criterion, the total number of patients eligible for the study was 96,855 . This study was approved by the Institutional Review Board of Kangbuk Samsung Hospital (IRB No. KBSMC 2017-10-015), and the requirement for informed consent was waived because we used de-identified retrospective data routinely collected during the health screening process.

\section{Data collection}

Baseline and follow-up examinations were conducted at Kangbuk Samsung Hospital Health Screening Center clinics in Seoul and Suwon. All participants were asked to complete a standardized, self-administered questionnaire about their socioeconomic status, smoking status, alcohol intake, regular exercise habits, medical history and medication use. Current alcohol use was assessed regarding as the frequency of alcohol drinking per week and amount of alcohol consumed per drinking day. ${ }^{31}$ Alcohol consumption was categorized into none, $<20 \mathrm{~g}$ of ethanol/day, and $\geq 20 \mathrm{~g}$ of ethanol/day for men and none, $<10 \mathrm{~g} / \mathrm{day}$, and $\geq 10 \mathrm{~g}$ of ethanol/day for women. ${ }^{32}$ Regular exercise habits were evaluated the weekly frequency of moderate- or vigorous-intensity physical activity. Smoking status was categorized into never, former, and current smokers. The pack-years was calculated as the number of cigarettes smoked per day multiplied by the number of years of previous or current smoking.

Weight, height, and sitting blood pressure were assessed by trained nurses. Weight was measured in light clothing and no shoes to the nearest $0.1 \mathrm{~kg}$ and height was measured to the nearest $0.1 \mathrm{~cm}$ using a digital scale. Body mass index (BMI) was calculated as weight in $\mathrm{kg}$ divided square of height and obesity was defined as BMI $\geq 25 \mathrm{~kg} / \mathrm{m}^{2}$ according to Asian-specific criteria. ${ }^{33,34}$ Hypertension was defined as a systolic blood pressure $\geq 140 \mathrm{mmHg}$, diastolic blood pressure $\geq 90 \mathrm{mmHg}$, or current use of antihypertensive medication.

Incident thyroid cancer cases

Thyroid cancer cases were primarily ascertained based on selfreport of physician-diagnosed thyroid cancer by standardized, self-administered questionnaires at the health screening visits. Thyroid cancer cases were defined as participants who reported physician-diagnosed thyroid cancer during follow-up. In a subsample of 57,741 participants who underwent health examination and provided informed consent for linkage to the national registries between 2011 and 2014, we evaluated the accuracy of self-reported physician-diagnosed thyroid cancer. When selfreported thyroid cancer was compared with confirmed thyroid cancer through national cancer registry data among 57,741 participants, the sensitivity and specificity for self-report of physician-diagnosed thyroid cancer were $98.1 \%$ and $99.8 \%$, respectively. Specific histologic types of thyroid cancer included papillary (97.4\%), follicular (1.4\%), carcinoid (0.1\%), medullary (0.1 \%), mixed medullary-papillary carcinoma (0.9\%), and unspecified cancer (0.2\%).

\section{Laboratory analyses}

Blood samples were taken from the antecubital vein after at least a 10-h fast. Measurement of serum uric acid, glucose, total cholesterol, triglyceride low-density lipoprotein cholesterol $(\mathrm{LDL}-\mathrm{C})$, alanine aminotransferase, aspartate aminotransferase levels, $\gamma$-glutamyl transferase, insulin, and high sensitivity C-reactive protein (hsCRP) has been described in detail elsewhere. $^{35}$ Insulin resistance was assessed with HOMA-IR according to the following equation: (fasting blood insulin $(\mu \mathrm{U} / \mathrm{ml}) \times$ fasting blood glucose $(\mathrm{mmol} / \mathrm{l})) / 22.5 .^{36}$ To assess thyroid function, serum TSH was measured by radioimmunoassay using a commercial kit (RIA-gnost VRhTSH, Schering-Cis Bio International, Gif-sur-Yvette, France), with a lower detection limit of $0.025 \mu \mathrm{lU} / \mathrm{ml}$. The normal range was $0.25-5.0 \mu \mathrm{lU} / \mathrm{ml}$, and the intra- and inter-assay coefficients of variation for quality control specimens were 1.2-5.7 and $2.4-5.4 \%$, respectively, for TSH. Diabetes was defined as a fasting serum glucose $\geq 126 \mathrm{mg} / \mathrm{dl}$, or current use of insulin or antidiabetic medications. The Laboratory Medicine Department at Kangbuk Samsung Hospital, Korea has been accredited by the Korean Society of Laboratory Medicine (KSLM) and the Korean Association of Quality Assurance for Clinical Laboratories (KAQACL). The laboratory participates in the College of American Pathologists (CAP) survey proficiency testing.

\section{Statistical analysis}

Subject characteristics were presented according to smoking status (never, former and current smokers) and were compared according to the smoking status using a linear regression test for continuous variables or $a X^{2}$ test for categorical variables. To test for linear trends, category numbers were used as continuous variables in regression models. All analyses were performed separately in men and women, as the association between smoking and thyroid cancer differs significantly by sex.

The study endpoint was the development of thyroid cancer. Follow-up for each participant extended from the baseline exam until either the development of thyroid cancer or the last health exam conducted prior to 31 December 2014. Incidence rate was calculated as the number of incident cases divided by personyears of follow-up. Since we knew that thyroid cancer had occurred at some point between the two visits but did not know the precise timing of development, we used a parametric proportional hazards model to take this type of interval censoring into account (stpm command in Stata). ${ }^{37}$ In these models, the baseline hazard function was parameterized with restricted cubic splines in log time with four degrees of freedom. The adjusted hazard ratio (aHR) with a 95\% confidence interval $(\mathrm{Cl})$ for incident thyroid cancer was estimated separately in men and women. The model was initially adjusted for age and then further adjusted for center (Seoul and Suwon), year of screening exam, alcohol intake ( $\geq 20$ vs. $<20 \mathrm{~g}$ of ethanol/day), regular exercise ( $\geq 3 \mathrm{vs} .<3$ times/ week), and educational level ( $\geq$ college graduate vs. college graduate or below) in model 1. To evaluate whether the association between smoking and incident thyroid cancer was mediated by $\mathrm{BMI}$ and TSH on a priori grounds, we included these variables in a multivariable-adjusted model (model 2). The following criteria for mediation analysis were used: (1) the predictor of interest (smoking status) was significantly related to the mediator (BMI and TSH), (2) the mediator (BMI and TSH) was significantly related to the outcome (thyroid cancer), and (3) the addition of the mediator (BMI and TSH) to the model both attenuated the coefficient of the predictor and had a statistically significant mediation effect. Moreover, to evaluate the impact of 
change of smoking status, pack- years and covariates over followup, we conducted additional analyses introducing smoking status and other covariates as a time-varying covariate in the models.

We assessed the proportional hazards assumption by examining graphs of estimated log (-log) survival. The number of categories was used as a continuous variable and tested on each model to determine linear trends of incidence.

In addition, we performed stratified analysis in pre-specified subgroups defined by age ( $\geq 50$ vs. $<50$ years), alcohol intake (none, $<20 \mathrm{vs}$. $\geq 20 \mathrm{~g}$ of ethanol/day for men; none, $<10 \mathrm{vs.} \geq 10 \mathrm{~g}$ for women), vigorous exercise (none, $<3$ vs. $\geq 3$ times/week), BMl $\left(\geq 25\right.$ vs. $\left.<25 \mathrm{~kg} / \mathrm{m}^{2}\right)$, HOMA-IR ( $\geq 2.5$ vs. $\left.<2.5\right)$, and hsCRP ( $\geq 1.0$ vs. $<1.0 \mathrm{mg} / \mathrm{L}$ ).

We conducted a series of sensitivity analyses to test the robustness of our primary results. First, Sensitivity analysis was conducted after implementing a 3-year wash-out period, during which any thyroid cancer cases identified were excluded. This approach was taken to assess if the original results may have been influenced by reverse causality in which thyroid cancer was present, but undiagnosed. Second, we performed the sensitivity analysis among 57,741 participants with available information on the cancer registry data. For this analysis, thyroid cancer cases were defined as participants who were diagnosed with a first primary thyroid malignant neoplasm during follow-up. Third, we performed the sensitivity analysis among 57,741 participants with available information on the cancer registry data using only papillary thyroid carcinoma as an endpoint because papillary thyroid carcinomas and medullary thyroid carcinomas have been considered different in terms of their incident rates, cell origins, risk factors and histopathological features. ${ }^{38}$

All statistical analyses were performed using STATA version 13.0 software (Stata Corp LP, College Station, TX).

\section{RESULTS}

Out of 96,855 subjects, $57.9 \%$ were male. The mean (SD) age of male and female participants were 40.4 (7.7) and 39.4 (7.7), respectively. Baseline characteristics of the subjects are shown by sex (Tables 1 and 2). Among male participants, current smokers were more likely to be obese, to drink alcohol, and have higher levels of LDL-C, triglycerides, and hsCRP and lower levels of systolic BP than never or ex-smokers (Table 1). Among female participants, the mean (SD) age and BMI was $39.4(7.7)$ and $22.0(2.9) \mathrm{kg} / \mathrm{m}^{2}$ respectively. Female current smokers were more likely to drink alcohol, to have higher levels of HDL-C and triglycerides, and to have lower levels of systolic BP, glucose, and LDL-C (Table 2).

Table 3 shows the development of thyroid cancer by smoking status. During 511,052.9 person-years of follow-up, 1,250 participants developed thyroid cancer (incidence rate, 3.4 per 1,000 person-years). The median follow-up period for participants was 5.9 years (interquartile range 4.0-6.8). For men, smoking status was significantly associated with a decreased risk of thyroid cancer. In the model adjusted for age, center, year of screening exam, alcohol intake, regular exercise, and educational level, the multivariable-adjusted HRs (95\% Cls) for incident thyroid cancer comparing current and former smokers to never smokers were $0.58(0.45-0.75)$ and $0.93(0.73-1.18)$, respectively. To explore whether the association between smoking status and development of thyroid cancer was mediated by BMI and TSH, we performed additional analyses adjusting for BMI and TSH. The association between smoking and incident thyroid cancer was slightly attenuated, but remained statistically significant in men ( $p$ for trend $<0.001$ ). In women, current smokers were less likely to develop thyroid cancer, but this association did not reach statistical significance; the multivariable-adjusted HRs (95\% Cls) for incident thyroid cancer comparing current and former smokers to never smokers were $0.64(0.33-1.23)$ and $0.72(0.46-1.14)$, respectively. When the association between smoking status and incident thyroid cancer was evaluated after introducing smoking status and confounders during follow-up as time-varying covariates, the inverse association between current smoking and incident thyroid cancer was still observed in time-dependent models in men but not in women.

We also examined the association between pack-years and the risk of thyroid cancer (Table 4). For men, there was a clear doseresponse relationship between smoking amount and thyroid cancer incidence ( $p$ for trend <0.001). In model 1, the multivariable-adjusted HRs ( $95 \% \mathrm{Cls})$ for incident thyroid cancer comparing $<10,10-19.9$, and $\geq 20$ pack-years with zero pack-years were 0.60 (0.41-0.88), 0.73 (0.54-0.99), and 0.44 (0.29-0.65), respectively. For women, the multivariable-adjusted HRs (95\% Cls) for incident thyroid cancer comparing $<5,5-9.9$, and $\geq 10$ pack-years with zero pack-years were $0.33(0.13-0.88), 0.91$ (0.30-2.04), and 1.39 (0.57-3.38), respectively. In mediation analysis after adjustment for BMI and TSH, these associations were similarly observed. When pack-years and confounders during follow-up were updated as time-varying covariates, the inverse association between pack-years and the risk of thyroid cancer was still observed in time-dependent models in men. In sensitivity analysis after excluding subjects who developed thyroid cancer within the first 3 years of follow-up, the associations of smoking status and pack-years with incident thyroid cancer were essentially unchanged (Supplementary Tables 3 and 4).

We performed additional analysis among 57,741 participants with available information on the cancer registry data (Supplementary Tables 3 and 4). In analysis using thyroid cancer based on the national cancer registry data among 57,741 participants only who gave informed consent for the linkage, we found an inverse association between current smoking and thyroid carcinoma and dose-dependent relationship between pack-years and thyroid carcinoma in men. When study endpoint was defined as papillary thyroid carcinoma, these associations were essentially unchanged. For women, the inverse association between current smoking and thyroid carcinoma was observed but did not reach statistical significance.

In subgroup analyses (Supplementary Tables 1 and 2), the association between smoking status and incident thyroid cancer was similar across pre-specified subgroups with no significant interactions with age ( $<50$ vs. $\geq 50$ years), alcohol intake (none, $<20$ vs. $\geq 20 \mathrm{~g}$ of ethanol/day for men; none, $<10$ vs. $\geq 10 \mathrm{~g}$ for women), vigorous exercise (none, $<3$ vs. $\geq 3$ times/week), BMl $\left(<25 \mathrm{~kg} / \mathrm{m}^{2}\right.$ vs. $\left.\geq 25 \mathrm{~kg} / \mathrm{m}^{2}\right)$, HOMA-IR ( $\left.<2.5 \mathrm{vs} . \geq 2.5\right)$, or hsCRP ( $<1.0 \mathrm{mg} / \mathrm{L}$ vs. $\geq 1.0 \mathrm{mg} / \mathrm{L}$ ).

\section{DISCUSSION}

In this large cohort of 96,855 young and middle-aged population, current smoking at baseline was significantly associated with a decreased risk of incident thyroid cancer in men. We also observed a non-statistically significant inverse association between current smoking and incidence of thyroid cancer in women. A dose-response relationship was also found between pack-years and incident thyroid cancer in men. These associations persisted after adjustment for potential confounders, indicating that smoking is an independent risk factor for incident thyroid cancer. When we evaluated whether the association between smoking status and development of thyroid cancer was mediated by $\mathrm{BMI}$ and $\mathrm{TSH}$, which are proposed mediators of thyroid cancer development in previous studies, the inverse association was slightly attenuated but still observed. Thus, levels of TSH and BMI by smoking could not fully explain the association between current smoking and thyroid cancer.

A pooled analysis of case-control studies of thyroid cancer in 2003 found a reduced risk of thyroid cancer in ever smokers. ${ }^{11}$ The 


\begin{tabular}{|c|c|c|c|c|c|}
\hline Characteristics & Overall & Never smoker & Ex-smoker & Current smoker & $P$ for trend \\
\hline Number & 56,060 & 17,005 & 16,392 & 22,663 & $<0.001$ \\
\hline Age (years) ${ }^{a}$ & $40.4(7.7)$ & $39.8(8.1)$ & $42.2(8.0)$ & $39.7(6.8)$ & $<0.001$ \\
\hline BMI $\left(\mathrm{kg} / \mathrm{m}^{2}\right)$ & $24.5(2.8)$ & $24.2(2.8)$ & $24.6(2.7)$ & $24.6(2.9)$ & $<0.001$ \\
\hline Vigorous exercise $(\%)^{c}$ & 20.4 & 21.2 & 27.0 & 15.1 & $<0.001$ \\
\hline High education level (\%) ${ }^{d}$ & 97.9 & 97.1 & 96.8 & 99.3 & 0.212 \\
\hline Diabetes (\%) & 4.7 & 3.6 & 5.4 & 5.0 & $<0.001$ \\
\hline Hypertension (\%) & 20.4 & 19.6 & 24.2 & 18.4 & $<0.001$ \\
\hline Systolic BP $(\mathrm{mmHg})^{a}$ & $118.5(12.9)$ & $118.7(13.0)$ & $119.5(13.1)$ & $117.6(12.6)$ & $<0.001$ \\
\hline LDL-C (mg/dL) ${ }^{a}$ & $115.7(29.1)$ & $114.7(28.4)$ & $116.0(29.0)$ & $116.3(29.7)$ & $<0.001$ \\
\hline $\mathrm{HDL}-\mathrm{C}(\mathrm{mg} / \mathrm{dL})^{\mathrm{a}}$ & $49.4(10.5)$ & $49.8(10.5)$ & $50.1(10.6)$ & $48.7(10.3)$ & $<0.001$ \\
\hline Triglycerides $(\mathrm{mg} / \mathrm{dL})^{\mathrm{e}}$ & $124(88-177)$ & $111(80-157)$ & $121(87-172)$ & 137 (97-195) & $<0.001$ \\
\hline HOMA-IR & $1.93(1.47-2.55)$ & $1.92(1.47-2.53)$ & $1.97(1.51-2.58)$ & $1.91(1.44-2.55)$ & 0.054 \\
\hline $\mathrm{hsCRP}(\mathrm{mg} / \mathrm{L})^{\mathrm{e}}$ & $0.6(0.3-1.1)$ & $0.5(0.3-1.0)$ & $0.5(0.3-1.1)$ & $0.6(0.3-1.2)$ & $<0.001$ \\
\hline
\end{tabular}

relationship was more pronounced in current smokers than former smokers, with odds ratios $(95 \% \mathrm{Cls})$ of $0.6(0.6-0.7)$ in current smokers and $0.9(0.8-1.1)$ in former smokers However, a significant association between current smokers and decreased risk of thyroid cancer was observed in women but not in men. In a prospective cohort study of U.S. radiologic technologists followed from 1983 through 2006, current smoking was inversely associated with thyroid cancer risk in women (HR $0.54,95 \% \mathrm{Cl}$ $0.35-0.82$ in women and HR $0.31,95 \% \mathrm{Cl} 0.09-1.04$ in men). ${ }^{17}$ In another prospective cohort study of postmenopausal women enrolled in the Women's Health Initiative with a follow-up of 12.7 years, current smokers had a reduced risk for papillary thyroid cancer ( $\mathrm{HR} \quad 0.34,95 \% \mathrm{Cl} \quad 0.15-0.78)$ compared with never smokers. ${ }^{18}$ However, in the same study, because the number of current smokers among cases was small, no associations or trends were seen for amount smoked. In a nine-year prospective study carried out on Koreans in the national insurance program, the inverse association between current smoking and thyroid cancer risk was observed in men (HR 0.6, 95\% Cl 0.5-1.0), but did not reach statistical significance due to relatively small number of thyroid cancer case. ${ }^{22}$ A pooled analysis of five prospective studies in the United States demonstrated that current smokers had a reduced risk of thyroid cancer ( $\mathrm{HR} 0.68,95 \% \mathrm{Cl} 0.55-0.85$ ) compared to never smokers, but this association was not significant in men. ${ }^{19}$ In the same meta-analysis, greater smoking intensity, duration, and pack-years were associated with further risk reduction. The inconsistent findings across studies could be attributable to differences in study design, sample size, study population (age, ethnicity, and sex composition), control for potential confounding factors, measures of smoking status, smoking prevalence or incidence of thyroid cancer. Most studies reported that the inverse association between current smoking and thyroid cancer was statistically significant in women ${ }^{17,18}$ but not in men due to the relatively lower incidence in men than in women. ${ }^{22}$ The present large-scale cohort study demonstrated that current smoking decreased thyroid cancer risk among men in a dose-response manner. The effect of smoking on thyroid cancer might confounded by $\mathrm{BMI}$, and smokers may have lower BMl. ${ }^{25}$ However, the association was still observed after adjustment for measured BMI and other confounders. Furthermore, this association was observed even when changes in smoking status and confounders during follow-up were treated as time-varying covariates. For women, we found non-statistically significant inverse association between current smoking and incidence of thyroid cancer. Even though $42 \%$ of the study population was female, the prevalence of current smoking was much lower in females than in males ( 2 vs. $40 \%$ ), resulting in lack of power to detect an association between current smoking and thyroid cancer risk in women.

The mechanisms that link smoking and thyroid cancer are not fully understood, but several possibilities have been proposed. First, higher TSH values are associated with a higher frequency and more advanced stage of thyroid cancer, ${ }^{27}$ while smoking seems to induce changes in thyroid function tests, such as decreases in TSH and increases in thyroid hormones. Thus, previous studies explained that lower TSH and lower BMI in smokers might influence the risk and protective effect of tobacco on thyroid cancer, which might be mediated by decreasing the body weight and TSH levels of the smoker. ${ }^{8,19,25,39}$ However, in the present study, the inverse association between smoking and thyroid cancer was still observed even after adjustment for TSH and BMI. Another possible biological mechanism underlying the association between current smoking and thyroid cancer is the anti-estrogenic effect. ${ }^{40}$ Estrogen has been suggested as an associated etiology of thyroid cancer, to explain the higher incidence of this cancer in females than in males. ${ }^{7,11}$ Vladan et al. also reported that smoking was negatively associated with thyroid cancer in men, due to its ability to decrease TSH secretion and its innate anti-estrogenic effect. ${ }^{15}$ Additionally, nicotine might be involved in the protective effects of smoking. The activation of nicotine receptors on immune cells 
Table 2. Baseline characteristics of study participants by smoking status among women

\begin{tabular}{|c|c|c|c|c|c|}
\hline \multirow[t]{2}{*}{ Characteristics } & \multirow[b]{2}{*}{ Overall } & \multicolumn{3}{|l|}{ Smoking status } & \multirow[t]{2}{*}{$P$ for trenc } \\
\hline & & Never smoker & Ex-smoker & Current smoker & \\
\hline Number & 40,795 & 38,712 & 1278 & 805 & $<0.001$ \\
\hline Age $(\text { years })^{a}$ & $39.4(7.7)$ & $39.4(7.7)$ & $38.4(8.1)$ & $39.0(7.9)$ & $<0.001$ \\
\hline BMI $\left(\mathrm{kg} / \mathrm{m}^{2}\right)$ & $22.0(2.9)$ & $22.0(2.9)$ & $22.0(3.2)$ & $22.0(3.2)$ & 0.666 \\
\hline Obesity (\%) & 13.6 & 13.6 & 15.2 & 14.7 & 0.103 \\
\hline Alcohol intake (\%) & 1.3 & 1.0 & 3.3 & 13.6 & $<0.001$ \\
\hline Vigorous exercise $(\%)^{c}$ & 20.5 & 20.6 & 17.3 & 20.3 & 0.093 \\
\hline High education level (\%) ${ }^{d}$ & 98.2 & 98.0 & 100.0 & 100.0 & $<0.001$ \\
\hline Diabetes (\%) & 1.7 & 1.6 & 2.6 & 1.5 & 0.287 \\
\hline Hypertension (\%) & 6.8 & 6.8 & 6.0 & 8.1 & 0.572 \\
\hline Systolic BP $(\mathrm{mmHg})^{\mathrm{a}}$ & $107.0(12.7)$ & $107.1(12.7)$ & $105.8(12.3)$ & $105.5(13.2)$ & $<0.001$ \\
\hline Diastolic BP $(\mathrm{mmHg})^{a}$ & $68.4(8.9)$ & $68.5(8.9)$ & $67.8(8.8)$ & $67.8(8.9)$ & 0.002 \\
\hline Glucose $(\mathrm{mg} / \mathrm{dL})^{\mathrm{a}}$ & $92.1(11.7)$ & $92.2(11.7)$ & $91.7(13.3)$ & $91.4(11.6)$ & 0.015 \\
\hline Total cholesterol $(\mathrm{mg} / \mathrm{dL})^{\mathrm{a}}$ & $184.5(32.1)$ & 184.5 & $184.4(32.8)$ & $183.7(31.0)$ & 0.493 \\
\hline LDL-C (mg/dL) $)^{a}$ & $101.7(27.5)$ & $101.8(27.5)$ & $100.0(27.6)$ & $99.0(26.8)$ & $<0.001$ \\
\hline $\mathrm{HDL}-\mathrm{C}(\mathrm{mg} / \mathrm{dL})^{\mathrm{a}}$ & $58.9(12.9)$ & $58.8(12.8)$ & $60.4(13.6)$ & $61.1(14.4)$ & $<0.001$ \\
\hline Triglycerides $(\mathrm{mg} / \mathrm{dL})^{\mathrm{e}}$ & $77(58-106)$ & $77(58-106)$ & $75.5(58-108)$ & $83(63-116)$ & $<0.001$ \\
\hline HOMA-IR & $1.73(1.26-2.26)$ & $1.73(1.26-2.26)$ & $1.68(1.21-2.23)$ & $1.71(1.37-2.23)$ & 0.941 \\
\hline $\mathrm{hsCRP}(\mathrm{mg} / \mathrm{L})^{\mathrm{e}}$ & $0.3(0.1-0.7)$ & $0.3(0.1-0.7)$ & $0.3(0.1-0.8)$ & $0.3(0.1-0.7$ & 0.067 \\
\hline
\end{tabular}

Data are presented as the mean (standard deviation), median (interquartile range), or percentage $B M I$ body mass index, $B P$ blood pressure, $L D L-C$ low-density lipoprotein-cholesterol, HDL-C high-density lipoprotein-cholesterol, HOMA-IR homeostasis model assessment of insulin resistance, $h s C R P$ high sensitivity Creactive protein. ${ }^{a}$ Mean (standard deviation) ${ }^{b} \geq 10 \mathrm{~g}$ of ethanol per day ${ }^{c} \geq 3$ times per week ${ }^{d} \geq C$ ollege graduate ${ }^{e}$ Median (interquartile range)

reduced the risk of Hashimoto's thyroiditis by shifting the autoimmune profile from pathogenic Th1 and Th17 responses to protective Th2 responses. ${ }^{25}$ The tobacco alkaloid anatabine, which has a structure similar to nicotine, also has been shown to decrease the incidence and severity of experimental autoimmune thyroiditis induced by thyroglobulin. ${ }^{41}$ Malignant transformation in the thyroid gland might be caused by cellular mediators produced by immune cells in states of chronic inflammation, ${ }^{42}$ and autoimmune thyroiditis such as Hashimoto's disease was reported to be associated with thyroid cancer including papillary carcinoma and thyroid lymphoma. ${ }^{43,44}$ Further mechanistic studies are warranted to elucidate the mechanism underlying the protective effect of smoking in thyroid cancer while considering chemical composition of cigarettes such as nicotine and anatabine.

Major strengths of our study include the large sample size, which allowed us to evaluate associations among stratified subgroups, and its prospective cohort study design, which eliminates the possibility of recall bias. Data collection in the Kangbuk Samsung Health Study is standardized carefully and subject to quality control. Our study population is a relatively young, middle aged, and healthy group who are less likely to be affected by biases related to comorbidities, compared to prior studies in older and higher risk populations.

We note a few limitations that should be considered. First, thyroid cancer was primarily ascertained on self-report of physician-diagnosed thyroid cancer. In our study, the accuracy of self-reported thyroid cancer was high when it was compared with confirmed thyroid cancer from national cancer registry data, with a sensitivity of $98.1 \%$ and a specificity of $99.8 \%$. Additionally, in sensitivity analysis using thyroid cancer based on the national cancer registry data, the results were similar. However, we cannot exclude the potential for measurement error in these variables, which might have resulted in some degree of residual confounding. Second, the number of current smokers in women was small and insufficient to reach a conclusion. We found that current smokers had a lower risk of thyroid cancer than never smokers in women and that the risk was gradually decreased by smoking status as a trend, but it was not statistically significant. It also may be due to the relatively low rate of female smokers in Korea or because smoking status in women was underestimated by the self-reported questionnaire. Generally, women tend to under-report smoking status, and there was a sex difference in self-reported and cotinine-verified smoking prevalence rates in Korea. ${ }^{45}$ While this is a limitation, to the best of our knowledge, this cohort study is one of the few studies to evaluate the association between current smoking and thyroid cancer in men with a large sample size, whereas most studies only have female subjects and a relatively lower number of men. Third, lifestyle variables (i.e., smoking, alcohol intake, and physical history) were collected based on a self-administered structured questionnaire used in the health checkup program in Korea as a part of the National Health Insurance plan. We cannot exclude that measurement error in these variables, which might have resulted in some degree of residual confounding. Fourth, number of years since quitting in former smokers was not available, limiting our ability to examine the role of former smoking on thyroid cancer. Finally, our study data included apparently healthy young and middle-aged, educated Koreans. We compared our subjects with a representative sample of the general Korean population (the Korea National Health and Nutrition Examination Survey, KNHANES). The age and sex standardization was performed using the direct method to the age structure of the Korean population aged $20-80$ in the year 2010 . The age and sex-standardized prevalence of type 2 diabetes (defined as fasting serum glucose level of $126 \mathrm{mg} / \mathrm{dL}$ or greater or the use of blood glucose lowering agents), hypertension, obesity (BMI $\geq$ $\left.25 \mathrm{~kg} / \mathrm{m}^{2}\right)$, and current smoker was lower than that of the general population (6.4 vs. $10.5 \%, 23.7$ vs. $29.1 \%, 28.4$ vs. $31.5 \%$, and 18.0 vs. $26.5 \%$, respectively). ${ }^{46}$ Therefore, it is highly probable that our study subjects were healthier than the general Korean 

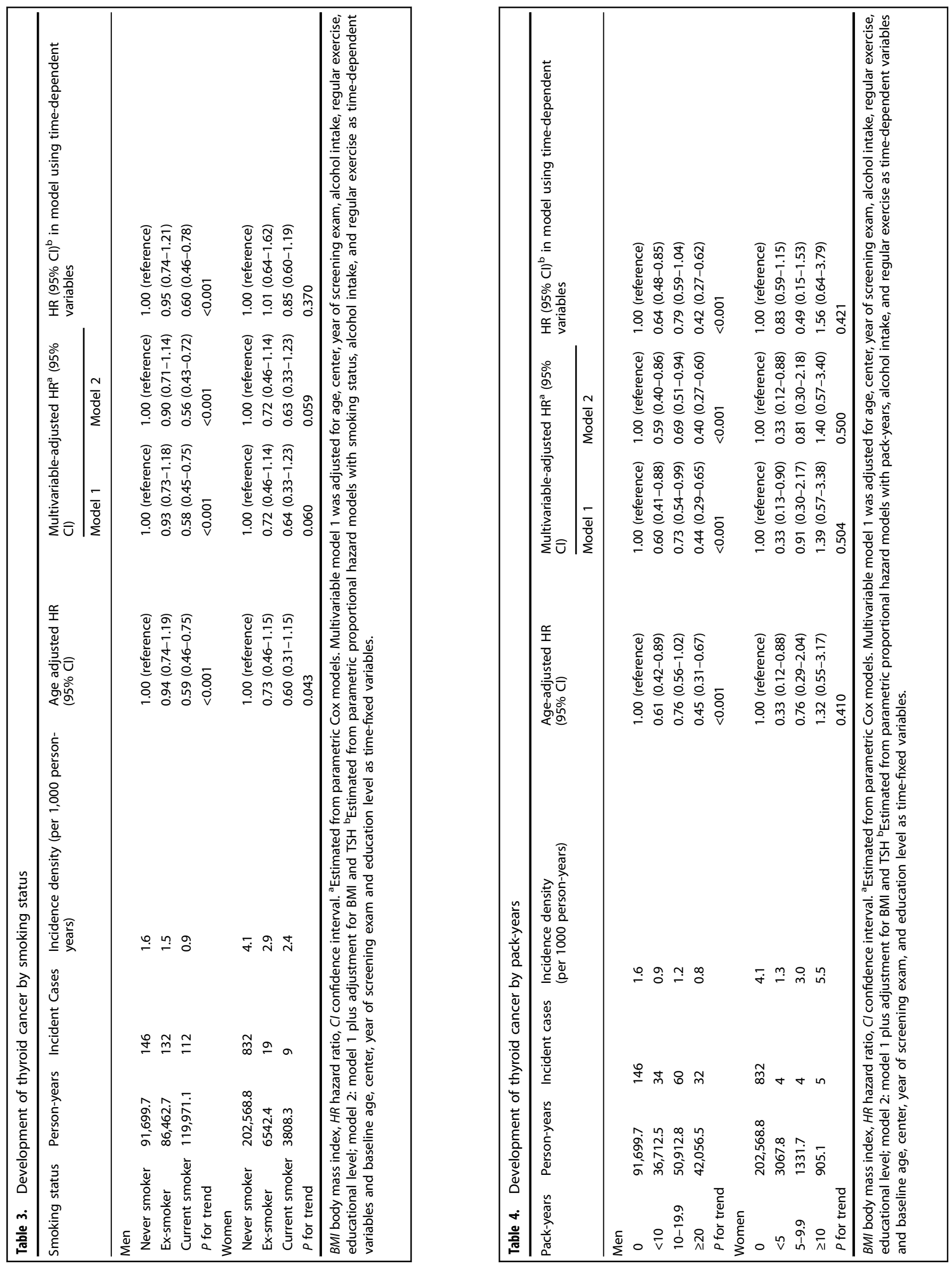
population. Our findings might not be generalizable to other populations with different characteristics of age and race/ ethnicity.

In conclusion, in this prospective cohort study, current smoking was associated with a reduced risk of thyroid cancer in Korean men. Current smoking and/or having high pack-years of exposure was associated with a reduced risk of thyroid cancer even after adjusting for TSH and BMI levels as potential mediators, indicating that the association between current smoking and development of thyroid cancer may not fully elucidated by TSH and BMI levels. Further mechanistic studies underlying the inverse association between smoking and thyroid cancer will help to understand the pathogenesis of thyroid cancer. However, our findings should not be interpreted as indicating that smoking can be recommended to prevent the development of thyroid cancer because smoking is still a major risk factor for various types of cancer and poor prognosis.

\section{ACKNOWLEDGEMENTS}

We acknowledge the dedicated efforts of investigators and staff at the Kangbuk Samsung Hospital Total Healthcare Screening Center.

\section{AUTHORS' CONTRIBUTIONS}

A.C., Y.C., H.S. and S.R. participated in study conception and design. J.A. and S.R. performed the data analysis. A.C., Y.C. and S.R. wrote the first draft of the manuscript and contributed to interpretation of data and critical revision of the manuscript for important intellectual content. All authors contributed to additional data interpretation and revisions and approval of the manuscript.

\section{ADDITIONAL INFORMATION}

Supplementary information is available for this paper at https://doi.org/10.1038/ s41416-018-0224-5.

Competing interests: The authors declare no competing interests.

Funding: The authors have no sources of study funding to declare.

Data availability: The data are not available to be shared publicly because we do not have a permission from the IRB to distribute the data. However, datasets generated during and/or analyzed during the current study are available from the Kangbuk Samsung Health Study through the corresponding author on reasonable request.

Ethics approval and consent to participate: This study was approved by the Institutional Review Board of Kangbuk Samsung Hospital (IRB No. KBSMC 2017-10015), and the requirement for informed consent was waived because we used deidentified retrospective data routinely collected during the health screening process. The study was performed in accordance with the Declaration of Helsinki.

Note: This work is published under the standard license to publish agreement. After 12 months the work will become freely available and the license terms will switch to a Creative Commons Attribution-NonCommercial-Share Alike 4.0 Unported License.)

\section{REFERENCES}

1. Pellegriti, G., Frasca, F., Regalbuto, C., Squatrito, S. \& Vigneri, R. Worldwide increasing incidence of thyroid cancer: update on epidemiology and risk factors. J. Cancer Epidemiol. 2013, 965212 (2013).

2. Stewart B. W., Wild C. World Cancer Report 2014. International Agency for Research on Cancer. World Health Organization. 2014;505.

3. Kitahara, C. M. \& Sosa, J. A. The changing incidence of thyroid cancer. Nat. Rev. Endocrinol. 12, 646-653 (2016).

4. Vigneri, R., Malandrino, P. \& Vigneri, P. The changing epidemiology of thyroid cancer: why is incidence increasing? Curr. Opin. Oncol. 27, 1-7 (2015).
5. Abualula N. A., Rodan M. F., Milligan R. A., Jacobsen K. H. Self-rated health among American adolescents with type 1 diabetes in the T1D Exchange Clinic Registry. J Diabetes Complications. 2018;32(1):83-8.

6. Hallquist, A., Hardell, L., Degerman, A. \& Boquist, L. Occupational exposures and thyroid cancer: results of a case-control study. Eur. J. Cancer Prev. 2, 345-349 (1993).

7. Galanti, M. R. et al. Reproductive history and cigarette smoking as risk factors for thyroid cancer in women: a population-based case-control study. Cancer epidemiology, biomarkers \& prevention: a publication of the American Association for Cancer Research, cosponsored by the American Society of Preventive. Oncology 5, 425-431 (1996).

8. KreigerN., \& ParkesR. Cigarette smoking and the risk of thyroid cancer. Eur. J. Cancer 36, 1973-1969 (2000).

9. Rossing, M. A., Cushing, K. L., Voigt, L. F., Wicklund, K. G. \& Daling, J. R. Risk of papillary thyroid cancer in women in relation to smoking and alcohol consumption. Epidemiology 11, 49-54 (2000).

10. Mack, W. J., Preston-Martin, S., Bernstein, L. \& Qian, D. Lifestyle and other risk factors for thyroid cancer in Los Angeles County females. Ann. Epidemiol. 12, 395-401 (2002).

11. Mack, W. J. et al. A pooled analysis of case-control studies of thyroid cancer: cigarette smoking and consumption of alcohol, coffee, and tea. Cancer Causes Control 14, 773-785 (2003).

12. Zivaljevic, V. et al. Cigarette smoking as a risk factor for cancer of the thyroid in women. Tumori 90, 273-275 (2004).

13. Guignard, R., Truong, T., Rougier, Y., Baron-Dubourdieu, D. \& Guenel, P. Alcohol drinking, tobacco smoking, and anthropometric characteristics as risk factors for thyroid cancer: a countrywide case-control study in New Caledonia. Am. J. Epidemiol. 166, 1140-1149 (2007).

14. Nagano, J. et al. A case-control study in Hiroshima and Nagasaki examining nonradiation risk factors for thyroid cancer. J. Epidemiol. 17, 76-85 (2007).

15. Zivaljevic, V. et al. Risk factors for well-differentiated thyroid cancer in men. Tumori 99, 458-462 (2013).

16. Myung, S. K., Lee, C. W., Lee, J., Kim, J. \& Kim, H. S. Risk factors for thyroid cancer: a hospital-based case-control study in Korean adults. Cancer Res. Treat. 49, 70-78 (2017).

17. Meinhold, C. L. et al. Nonradiation risk factors for thyroid cancer in the US Radiologic Technologists Study. Am. J. Epidemiol. 171, 242-252 (2010).

18. Kabat, G. C., Kim, M. Y., Wactawski-Wende, J. \& Rohan, T. E. Smoking and alcohol consumption in relation to risk of thyroid cancer in postmenopausal women. Cancer Epidemiol. 36, 335-340 (2012).

19. Kitahara, C. M. et al. Cigarette smoking, alcohol intake, and thyroid cancer risk: a pooled analysis of five prospective studies in the United States. Cancer Causes Control 23, 1615-1624 (2012).

20. Blakely, T. et al. The association of active smoking with multiple cancers: national census-cancer registry cohorts with quantitative bias analysis. Cancer Causes Control 24, 1243-1255 (2013).

21. Iribarren, C., Haselkorn, T., Tekawa, I. S. \& Friedman, G. D. Cohort study of thyroid cancer in a San Francisco Bay area population. Int. J. Cancer 93, 745-750 (2001).

22. Jee, S. H., Samet, J. M., Ohrr, H., Kim, J. H. \& Kim, I. S. Smoking and cancer risk in Korean men and women. Cancer Causes Control 15, 341-348 (2004).

23. Navarro Silvera, S. A., Miller, A. B. \& Rohan, T. E. Risk factors for thyroid cancer: a prospective cohort study. Int. J. Cancer 116, 433-438 (2005).

24. Cho, Y. A. \& Kim, J. Thyroid cancer risk and smoking status: a meta-analysis. Cancer Causes Control 25, 1187-1195 (2014).

25. Wiersinga, W. M. Smoking and thyroid. Clin. Endocrinol. 79, 145-151 (2013).

26. Winslow, U. C., Rode, L. \& Nordestgaard, B. G. High tobacco consumption lowers body weight: a Mendelian randomization study of the Copenhagen General Population Study. Int J. Epidemiol. 44, 540-550 (2015).

27. Boelaert, K. The association between serum TSH concentration and thyroid cancer. Endocr. -Relat. Cancer 16, 1065-1072 (2009).

28. Soldin, O. P., Goughenour, B. E., Gilbert, S. Z., Landy, H. J. \& Soldin, S. J. Thyroid hormone levels associated with active and passive cigarette smoking. Thyroid 19, 817-823 (2009)

29. Chang, Y. et al. Metabolically healthy obesity and development of chronic kidney disease: a cohort study. Ann. Intern. Med. 164, 305-312 (2016).

30. Zhang, Y. et al. Thyroid hormones and mortality risk in euthyroid individuals: the Kangbuk Samsung health study. J. Clin. Endocrinol. Metab. 99, 2467-2476 (2014).

31. Park, J. T., Kim, B. G. \& Jhun, H. J. Alcohol consumption and the CAGE questionnaire in Korean adults: results from the Second Korea National Health and Nutrition Examination Survey. J. Korean Med. Sci. 23, 199-206 (2008).

32. Fernandez-Sola, J. Cardiovascular risks and benefits of moderate and heavy alcohol consumption. Nat. Rev. Cardiol. 12, 576-587 (2015).

33. Wen, C. P. et al. Are Asians at greater mortality risks for being overweight than Caucasians? Redefining obesity for Asians. Public Health Nutr. 12, 497-506 (2009). 
34. World Health Organization, Regional Office for the Western Pacific. The AsiaPacific perspective: redefining obesity and its treatment (Sydney, Health Communications Australia, 2000).

35. Chang, Y. et al. Metabolically-healthy obesity and coronary artery calcification. $J$. Am. Coll. Cardiol. 63, 2679-2686 (2014).

36. Matthews, D. R. et al. Homeostasis model assessment: insulin resistance and betacell function from fasting plasma glucose and insulin concentrations in man. Diabetologia 28, 412-419 (1985).

37. Royston, P. \& Parmar, M. K. Flexible parametric proportional-hazards and proportional-odds models for censored survival data, with application to prognostic modelling and estimation of treatment effects. Stat. Med. 21, 2175-2197 (2002).

38. Maia, A. L., Wajner, S. M. \& Vargas, C. V. Advances and controversies in the management of medullary thyroid carcinoma. Curr. Opin. Oncol. 29, 25-32 (2017).

39. Asvold, B. O., Bjoro, T. \& Vatten, L. J. Association of serum TSH with high body mass differs between smokers and never-smokers. J. Clin. Endocrinol. Metab. 94, 5023-5027 (2009).
40. Derwahl, M. \& Nicula, D. Estrogen and its role in thyroid cancer. Endocr.-Relat. Cancer 21, T273-T283 (2014).

41. Caturegli, P. et al. Anatabine ameliorates experimental autoimmune thyroiditis Endocrinology 153, 4580-4587 (2012).

42. Balkwill, F. \& Mantovani, A. Inflammation and cancer: back to Virchow? Lancet 357, 539-545 (2001)

43. Resende de Paiva, C., Gronhoj, C., Feldt-Rasmussen, U. \& von Buchwald, C. Association between Hashimoto's Thyroiditis and Thyroid Cancer in 64,628 Patients. Front Oncol. 7, 53 (2017).

44. Noureldine, S. I. \& Tufano, R. P. Association of Hashimoto's thyroiditis and thyroid cancer. Curr. Opin. Oncol. 27, 21-25 (2015).

45. Jung-Choi, K. H., Khang, Y. H. \& Cho, H. J. Hidden female smokers in Asia: a comparison of self-reported with cotinine-verified smoking prevalence rates in representative national data from an Asian population. Tob. Control. 21, 536-542 (2012).

46. Korean Institute for Health and Social Affairs. Korea Health Statistics 2010: Korea National Health and Nutrition Examination Survey (KNHANES V-1), 2011. 